\title{
OP oder nicht, wat bruk wi denn nu?
}

\author{
Tobias Fehmer, Michael Hahn, Oliver Pieske
}

\begin{abstract}
Das 1. AOTrauma-Seminar Nord-West hat am 27. April 2018 erfolgreich im Alten Landtag Oldenburg (Oldenburg) unter der Leitung von Prof. Dr. Michael Hahn, Bremen, Dr. Tobias Fehmer, Sanderbusch und Priv.-Doz. Dr. Oliver Pieske, Oldenburg, mit 52 Teilnehmern stattgefunden.
\end{abstract}

\begin{abstract}
Den Organisatoren war es wichtig, in der Flächenregion mit mehr als 2 Millionen Einwohnern ein unfallchirurgisch-orthopädisches AOT-Seminar mit hochkarätiger Referentenbesetzung zu etablieren. Dabei sollte die Patientenversorgung im Mittelpunkt stehen mit der Frage: „operieren - ja oder nein - wat bruk wi denn nu“?
\end{abstract}

Der Altersdurchschnitt der Patienten in unfallchirurgisch/ orthopädischen Kliniken ist in den letzten Jahren dramatisch gestiegen. Wir sehen immer mehr Patienten, die, zusätzlich zu erlittenen altersbedingten Frakturen, multiple Nebenerkrankungen aufweisen. Der Abstimmung unserer therapeutischen Maßnahmen auf diese Patienten unter Berücksichtigung der Multimorbidität kommt eine entscheidende Bedeutung zu.

Neben den anatomischen Regionen wie Thorax, obere Extremität, Wirbelsäule/Becken und untere Extremität wur- den auch die präoperative Antibiotikaprophylaxe mit der Indikationsstellung zur Dekolonisation (Dr. Behnke, Anästhesist, Wittmund) sowie Zukunftsfragen zur Unfallchirurgie in Deutschland mit den Möglichkeiten der computerassistierten Visualisierung zur Diagnostik und intraoperativen Therapie berücksichtigt (Prof. Wolfgang Böcker, Ludwig-Maximilian-Universität München).

Darüber hinaus diskutierten das Auditorium und die 14 Faculty-Mitglieder an zahlreichen Fällen konkrete diagnostische und therapeutische Behandlungsstrategien.

Die Evaluation des Seminars konnte den Erfolg der Veranstaltung unterstreichen. Insbesondere erfuhren Referenten und Experten durch ihre Themenbegeisterung, freundliche Zugänglichkeit und persönliche Interaktion die Wertschätzung der Teilnehmer.

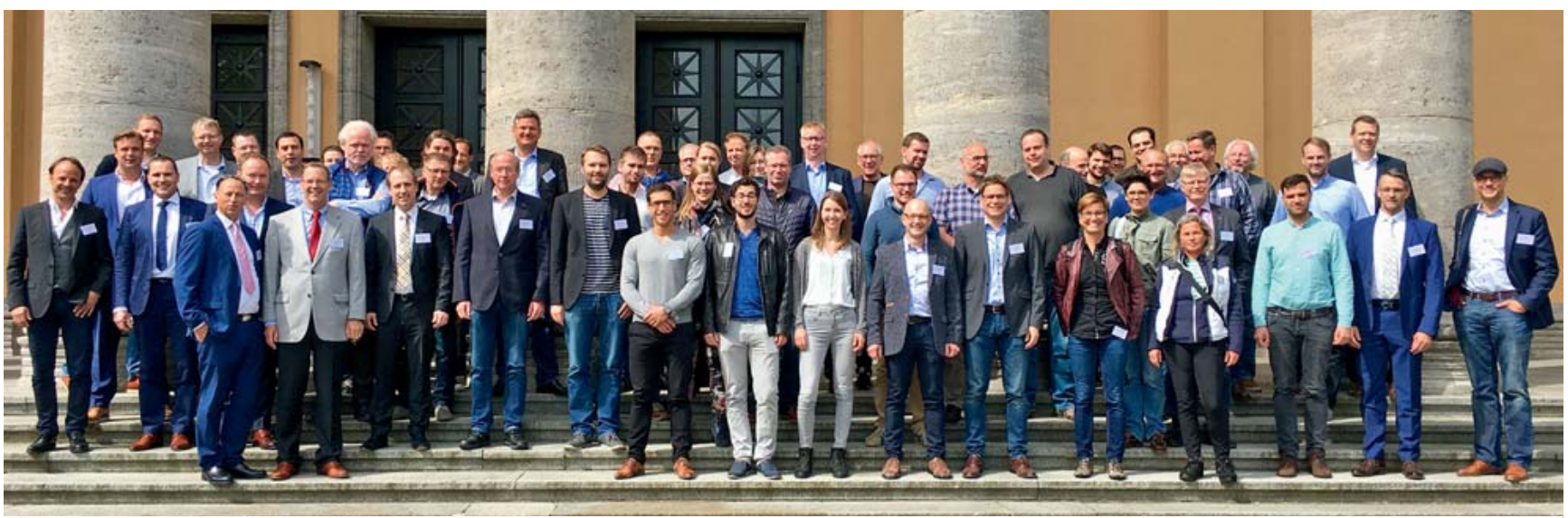

Abb. 1 Gruppenphoto. 


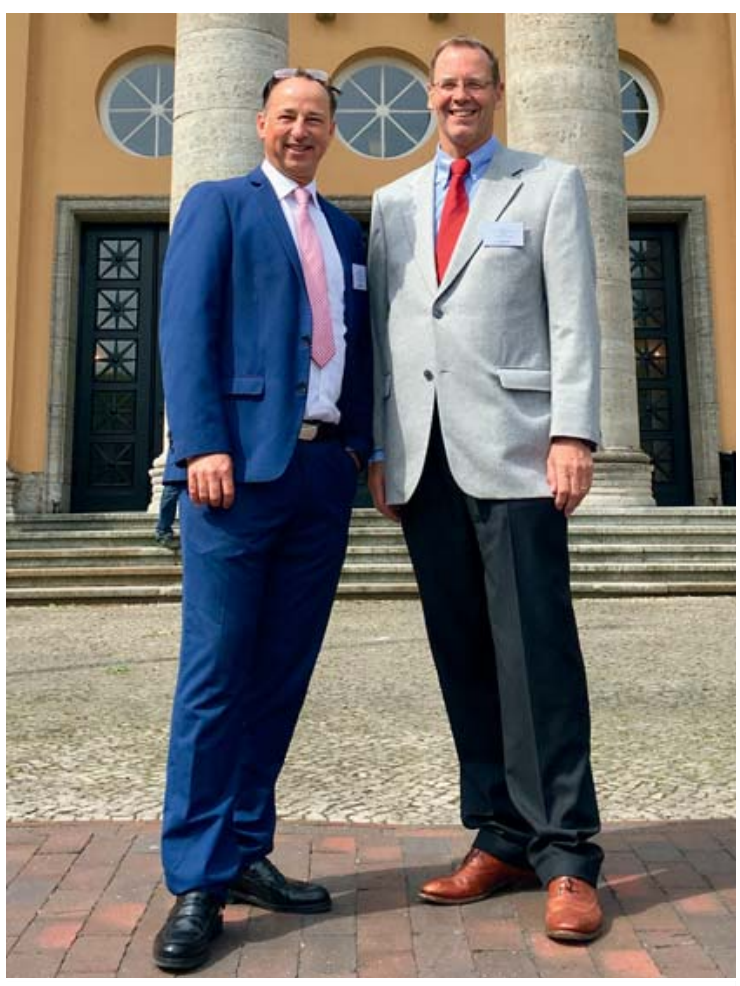

Abb. 2 Seminarleiter Pieske und Hahn.

Auch der Veranstaltungsort im Alten Landtag, eines im Auftrag des Großherzogtums Oldenburg errichteten Gebäudes, erfuhr eine äußerst positive Rückmeldung.

Im nächsten Jahr findet das AOTrauma-Seminar NordWest in Bremen und im übernächsten Jahr in Sanderbusch-Wilhelmshaven statt.

PD Dr. Oliver Pieske, Evangelisches Krankenhaus Oldenburg

E-Mail: oliver.pieske@evangelischeskrankenhaus.de

\section{Bibliografie}

DOI https://doi.org/10.1055/a-0647-2593

OP-JOURNAL 2018; 34: 188-189 @ Georg Thieme Verlag KG Stuttgart · New York ISSN 0178-1715 